\title{
Temblor ortostático como causa de inestabilidad
}

\author{
Raquel Manrique-Huarte ${ }^{\mathrm{a}, *}$, Juan Arcocha ${ }^{\mathrm{b}}$ y Nicolás Pérez-Fernández ${ }^{\mathrm{a}}$ \\ a Departamento de Otorrinolaringología, Clínica Universidad de Navarra. Pamplona, Navarra, España \\ b Departamento de Neurofisiología, Clínica Universidad de Navarra, Pamplona, Navarra, España
}

\author{
PALABRAS CLAVE \\ Inestabilidad; \\ Electromiografía; \\ Temblor ortostático
}

\section{KEYWORDS}

Instability;

Electromyography;

Orthostatic tremor

\begin{abstract}
Resumen El temblor ortostático (TO) es una enfermedad neurológica de origen desconocido caracterizada por un temblor de $10-20 \mathrm{~Hz}$ en las piernas en bipedestación. Se manifiesta por mareo e inestabilidad, que típicamente mejoran al apoyarse o sentarse y la ingesta de pequeñas cantidades de alcohol lo reduce de manera significativa. Se muestran 4 casos clínicos atendidos en nuestra consulta cuyo diagnóstico sugiere ser el de TO. Consideramos que ante un paciente con inestabilidad, es preciso plantearse como diagnóstico diferencial un TO. La historia clínica nos orienta hacia esta entidad y en caso de sospecha, el diagnóstico definitivo viene dado por el registro de la electromiografía en las extremidades inferiores en condición de reposo sentado y en ortostatismo donde se registra un temblor de 10-20 Hz. El tratamiento es médico y, se emplea el clonazepam como primera opción terapéutica.

๑ 2011 Elsevier España, S.L. Todos los derechos reservados.
\end{abstract}

\section{Orthostatic tremor inducing instability}

Abstract Orthostatic tremor (OT) is a neurological disease of unknown aetiology. It is defined by the presence of a $10-20 \mathrm{~Hz}$ tremor in the legs while standing still. Symptoms described are dizziness and instability that diminish if the patient sits down or leans on something; drinking small amounts of alcohol significantly reduces OT. Due to the dizziness and/or unsteadiness, these patients are usually referred to the neuro-otology department. We report 4 cases diagnosed with OT. The diagnosis of OT should be considered for patients with instability. The clinical history is a key factor to suspect this entity, and the diagnosis is given by the register of $10-20 \mathrm{~Hz}$ contractions on limb electromyography. Treatment for this disease consists of medical treatment; the first option is clonazepam.

(c) 2011 Elsevier España, S.L. All rights reserved.
* Autor para correspondencia.

Correo electrónico: rmanrique@unav.es (R. Manrique-Huarte).

\section{Introducción}

El temblor ortostático (TO) es una entidad rara, descrita por primera vez por Heilman ${ }^{1}$, de prevalencia desconocida debido a la falta de datos epidemiológicos ${ }^{2}$. Se caracteriza por registrarse una actividad electromiográfica intensa y regular de $10-20 \mathrm{~Hz}$, en la musculatura de las 
extremidades inferiores; si bien, un patrón característico en el registro electromiográfico con salvas $6-20 \mathrm{~Hz}$ es sugestivo de esta entidad ${ }^{3}$. Esto genera una sensación de inestabilidad, mareo y/o molestias en la parte posterior de las piernas durante la sedestación. Precisamente la alta frecuencia de contracción hace que no se perciba dicho temblor sino sus consecuencias entre las que destaca la inestabilidad ${ }^{4}$. Característicamente, ésta se intensifica al estar en bipedestación mientras que el sentarse, sujetarse a algo o deambular lo alivia; es necesaria la contracción isométrica de la musculatura inferior para que se desencadene el temblor ${ }^{5}$. La causa es desconocida y parece originarse en un generador supraespinal localizado en el tronco del encéfalo influenciado por la actividad descontrolada del circuito tálamo-cortical ${ }^{6}$. En el $75 \%$ de los casos es idiopático y en el $25 \%$ asocian alteraciones neurológicas adicionales ${ }^{7}$ que por frecuencia son parkinsonismo, síndrome de piernas inquietas, clonus, mioclonus y ataxia cerebelosa. Suele iniciarse hacia los 50 años, con un retraso diagnóstico promedio de 5-8 años y, en la mayoría de los casos los síntomas permanecen invariables; en el $15 \%$ de los pacientes los síntomas se extienden a tronco y brazos ${ }^{7}$.

Habitualmente los estudios de función vestibular son normales o inespecíficos, sin valor localizador. Para su diagnóstico, la posturografía (tanto estática como dinámica) permite hacer un primer cribado $^{8}$, el diagnóstico definitivo se realiza mediante el estudio electromiográfico (EMG) de la actividad en la musculatura gravitatoria y antigravitatoria de la pierna.

A pesar de ser una entidad poco conocida para el otorrinolaringólogo se debe incluir en el diagnóstico diferencial de pacientes con inestabilidad crónica, habituales en las consultas de otoneurología. Se presentan 4 casos que plantean también diferentes formas de presentarse el proceso.

\section{Métodos y resultados}

Presentamos una serie de 4 casos clínicos (tabla 1) de pacientes atendidos en la consulta de otorrinolaringología de un centro terciario que acuden por inestabilidad y cuya historia clínica nos hace sospechar un TO.

\section{Caso clínico 1}

Mujer de 65 años, sana, que consulta por inestabilidad, sensación nauseosa y malestar general, junto con pesadez en extremidades inferiores al estar quieta de pie y que se alivia al mover las piernas. Esta sintomatología se presenta de manera episódica una vez por semana desde hace dos años durante 3-4 horas.

La exploración otológica, así como la exploración otoneurológica (seguimiento ocular y sacadas, nistagmo espontáneo evocado con y sin fijación visual, reflejo vestíbulo-oculomotor, maniobra de impulso cefálico hacia derecha e izquierda, agudeza visual dinámica; nistagmo tras agitación cefálica; maniobra de Dix-Hallpike, nistagmo de posición) son normales. Maniobras de Romberg y Fukuda normales. Marcha en tandem normal. La exploración neurológica constata la ausencia de signos neurológicos asociados.

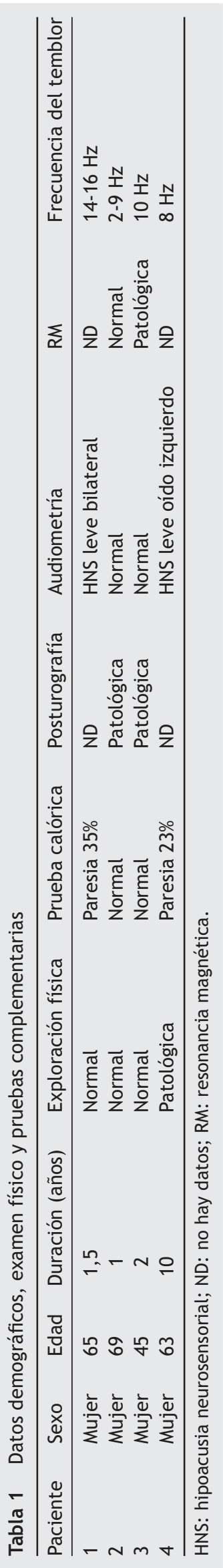




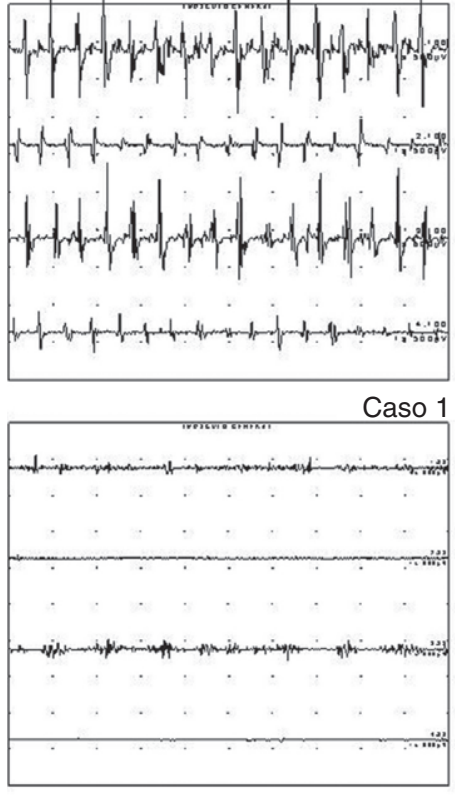

Caso 3

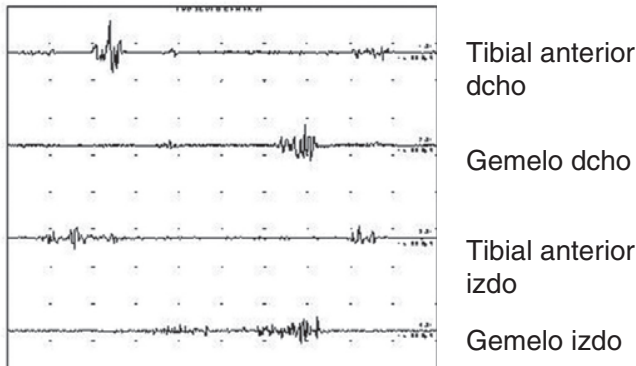

Caso 2

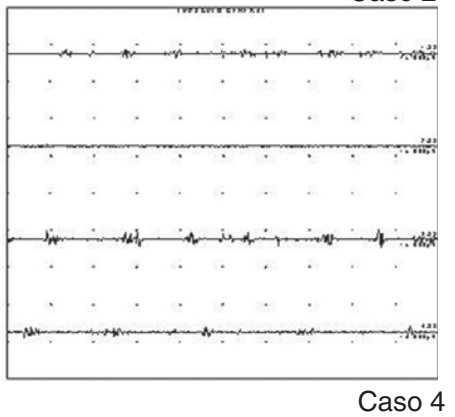

Figura 1 Registro del estudio electromiográfico de los 4 casos. Las gráficas representan un período de un segundo, las contracciones se presentan en salvas con el paciente en ortostatismo.

En la audiometría tonal se objetiva una hipoacusia neurosensorial leve simétrica bilateral. En la prueba calórica (con agua, bitérmica, monoaural y alternativa) se registra una paresia canalicular derecha del 35\%. El resultado en el estudio de los potenciales evocados miogénicos cervicales con estímulo acústico (PVEMac) fue normal (tabla 1). Se solicita PET sin observarse hallazgos patológicos.

La rehabilitación vestibular planteada en un primer momento empeoró la sintomatología.

El EMG muestra una actividad muscular rítmica de 14-16 Hz, con salvas de alta frecuencia de descarga de carácter alternante (fig. 1).

Se confirma el diagnóstico de sospecha de temblor ortostático y se deriva al Departamento de Neurología para iniciar tratamiento con clonazepam.

\section{Caso clínico 2}

Mujer de 69 años con antecedentes de hipertensión arterial (tratada con amlodipino y valsartán), dislipemia, hiperglucemia y sobrepeso que es derivada a la consulta de otoneurología por una «sensación de balanceo de delante a atrás» en ortostatismo que mejora con la marcha y con el consumo de pequeñas cantidades de bebidas alcohólicas. Ha sido diagnosticada de vértigo psicógeno.

La exploración otológica y otoneurológica son normales, así como la neurológica. La audiometría tonal, la videonistagmografía (VNG: registro del nistagmo y prueba calórica), PVEMac, así como la resonancia magnética (RM) cerebral son normales; la posturografía indica un patrón de preferencia visual (tabla 1).

El EMG muestra en ortostatismo unas descargas musculares de $2-9 \mathrm{~Hz}$, rítmicas y de alta frecuencia de descarga (fig. 1).
Se deriva al paciente al Departamento de Neurología para estudio e iniciar tratamiento con clonazepam.

\section{Caso clínico 3}

Paciente de 45 años sin antecedentes de interés, que presenta sensación continua de inestabilidad desde hace dos años. La paciente menciona que al estar en bipedestación, esta sintomatología empeora, no varía en ambientes muy iluminados ni oscuros y tampoco cuando las características de la superficie de apoyo son menos firmes. Dice que cuando peor se siente, el detenerse no le ayuda a mejorar, y que solo logra alivio al sentarse o sujetarse a algo. Las molestias las percibe inicialmente en las piernas y mejora con la ingesta ocasional de alcohol. No episodios de vértigo, no hipoacusia. Acúfeno bilateral que aumenta con la inestabilidad. Ha sido derivada al psiquiatra que no identifica alteraciones de interés.

La exploración otológica y otoneurológica es normal. La audiometría tonal, VNG y PVEMac son normales, así como la exploración neurológica.

La posturografía indica una inadecuada utilización de la información sensorial para el equilibrio con un patrón de déficit vestibular (tabla 1 ).

La RM en secuencias de difusión muestran una asimetría en la apreciación del haz córtico espinal y una reducción de volumen del pedúnculo cerebral del lado derecho.

El EMG muestra actividad a $10 \mathrm{~Hz}$, rítmicas y de alta frecuencia de descarga (fig. 1).

Se solicita valoración por neurología que establece el diagnóstico de TO, así como de una encefalopatía vascular leve secundaria a trombofilia congénita heterocigota factor $\checkmark$ Leiden. Inicia tratamiento con clonazepam. 
Tabla 2 Clasificación de patologías que cursan con temblor con su rango frecuencial y tipo de temblor según el Movement Disorder Society Consensus (1998)

\begin{tabular}{lcl}
\hline Diagnóstico & Rango frecuencial & Tipo de temblor \\
\hline Temblor fisiológico exagerado & $5-12 \mathrm{~Hz}$ & Postural \\
Temblor esencial & $5-10 \mathrm{~Hz}$ & Postural \\
Temblor ortostático & $10-20 \mathrm{~Hz}$ & Postural \\
Temblor postural y de acción & $6-11 \mathrm{~Hz}$ & Postural y acción \\
Distonía & $5-10 \mathrm{~Hz}$ & Postural y acción \\
Temblor parkinsoniano & $4-7 \mathrm{~Hz}$ & Reposo \\
Temblor cerebeloso & $3-5 \mathrm{~Hz}$ & Postural y acción \\
Temblor de Holmes & $2-5 \mathrm{~Hz}$ & Reposo y acción \\
Temblor palatino & $2-4 \mathrm{~Hz}$ & Reposo \\
Temblor neuropático & $5-11 \mathrm{~Hz}$ & Postural \\
Inducido por drogas y tóxicos & $4-11 \mathrm{~Hz}$ & Reposo, postural y/o acción \\
Psicógeno & $5-10 \mathrm{~Hz}$ & Reposo, postural y/o acción \\
\hline
\end{tabular}

\section{Caso clínico 4}

Mujer de 63 años con antecedentes médicos de migraña con aura que presenta inestabilidad y vértigo.

El primer episodio de vértigo lo relata hace 10 años; desde ese momento ha presentado varios episodios de horas a días de duración con acúfeno asociado en oído izquierdo. Además, la paciente refiere una sensación de inestabilidad en la deambulación más intensa cuando está de pie parada sin sujeción a nada y que es su síntoma más molesto por las limitaciones que impone.

La exploración otológica es normal. En la exploración otoneurológica destaca un nistagmo vertical hacia arriba y horario en la maniobra de Dix-Hallpike izquierda de muy leve intensidad. La exploración neurológica no muestra hallazgos patológicos de interés.

La audiometría tonal muestra una pérdida de $30 \mathrm{~dB}$ en oído izquierdo. En la prueba calórica (con agua, bitérmica, monoaural y alternativa) se registra una paresia canalicular derecha del 23\%. Los PVEMac son normales (tabla 1).

La palpación de extremidades inferiores muestra signos de fasciculaciones en ortostatismo. El EMG muestra salvas de $8 \mathrm{~Hz}$, rítmicas y alternantes (fig. 1).

Se diagnostica de vértigo posicional paroxístico benigno de canal semicircular posterior izquierdo con inestabilidad residual; ante la sospecha de TO se inicia tratamiento con clonazepam.

\section{Discusión}

Se han presentado 4 casos de TO cuyo motivo de consulta es la inestabilidad.

Ante un paciente con inestabilidad crónica (> 3 meses) el abordaje clínico debe ir orientado a: 1) identificar formas sindrómicas, 2) hacer un buen diagnóstico retrospectivo, 3) descartar alteraciones asociadas, 4) asegurarse que no es una alteración de la marcha, y 5) tener en cuenta que el tratamiento va a ser multidisciplinario. El diagnóstico diferencial incluye numerosas entidades pero la historia clínica ofrece importantes pistas para el diagnóstico. En los casos clínicos mostrados, el síntoma «diferente» de lo habitual es el empeoramiento en ortostatismo y mejoría en la deambulación que es contrario a lo que los pacientes con inestabilidad crónica secundaria a vestibulopatía refieren siempre. La necesidad de una mayor indagación viene dado porque la exploración vestibular es habitualmente normal salvo en aquellos casos con comorbilidades (como en nuestros casos 1 y 4 ).

El error diagnóstico más frecuente puede deberse a la falta de familiaridad con este proceso ${ }^{9}$ lo cual, dadas sus características atípicas, hace fácil diagnosticar al paciente como de inestabilidad psicógena una vez se ha descartado una enfermedad vestibular o sistémica. Desde el punto de vista neurológico el diagnóstico diferencial se debe hacer con otras enfermedades y trastornos del movimiento que pueden generar temblores ${ }^{10}$ (tabla 2).

Por ello, ante la sospecha es obligado solicitar un estudio electromiográfico específico que permite hacer el diagnóstico de certeza. La presencia de descargas musculares de breve duración desencadenadas por el ortostatismo en ambos miembros inferiores es el hallazgo característico en el TO. A diferencia de otros autores, que relatan la existencia de un alto grado de correlación entre posturografía estática y EMG ${ }^{11}$ no ha ocurrido así en nuestro caso. Esto quizás es debido a que hemos empleado un equipo de posturografía dinámica.

Las opciones terapéuticas son múltiples y deben ser indicadas por el neurólogo; habitualmente, el clonazepam es el fármaco de elección al inicio. Los 4 pacientes han seguido un curso satisfactorio con una mejoría evidente. Los efectos secundarios de la medicación fueron controlados cambiando la posología y toma cuando fue necesario.

En conclusión, el TO es una entidad clínica rara, cuya historia clínica es clave para sospechar esta afección. Para su diagnóstico, un estudio posturográfico analizando la respuesta en el dominio de la frecuencia (FFT) puede orientar al diagnóstico; si bien, el diagnóstico de certeza viene determinado por el registro EMG.

\section{Conflicto de intereses}

Los autores declaran no tener ningún conflicto de intereses. 


\section{Bibliografía}

1. Heilman KM. Orthostatic tremor. Arch Neurol. 1984;41:880-1.

2. Jones L, Bain P. Orthostatic tremor. Pract Neurol. 2011;11: 240-3.

3. Fitzerald PM, Jankovic J. Orthostatic tremor: an association with essential tremor. Mov Disord. 1991;6:60-4.

4. Karlberg M, Fransso PA, Magnusson M. Posturography can be used to screen for primary orthostatic tremor, a rare cause of dizziness. Otol Neurotol. 2005;26:1200-3.

5. Boroojerdi B, Ferbert A, Fottys H, Kosinski CM, Noth J, Schwarz M. Evidence for a non-orthostatic origin of orthostatic tremor. J Neurol Neurosurg Psychiatry. 1990;66:284-8.

6. Guridi J, Rodríguez-Oroz MC, Arbizu J, Alegre M, Prieto E, Landecho I, et al. Successful thalamic deep brain stimulation for orthostatic tremor. Mov Disord. 2008;23:1808-11.
7. Gerschlager W, Münchau A, Katzenschlager R, Brown P, Rothwell JC, Quinn N, et al. Natural history and syndromic associations of orthostatic tremor: a review of 41 patients. Mov Disord. 2004;19:788-95.

8. Bronstein AM, Guerraz M. Visual-vestibular control of posture and gait: physiological mechanisms and disorders. Curr Opin Neurol. 1999;12:5-11.

9. Mastain B, Cassin F, Guieu JD, Destee A. Primary orthostatic tremor. Rev Neurol (Paris). 1998;154:322-9.

10. Deuschl G, Bain P, Brin M. Ad hoc Scientific Committee. Consensus statement of the movement disorder Society on Tremor. Mov Disord. 1998;13 Suppl 3:S2-23.

11. Yarrow K, Brown P, Gresty MA, Bronstein A. Force platform recordings in the diagnosis of primary orthostatic tremor. Gait Posture. 2001;13:26-34. 